\title{
The Relationship Between Serum Albumin Level and Nutritional Parameters with Mortality Rate, For Icu-Covid-19 Patients at Hazem Mebaireek General Hospital (HMGH), State of Qatar (March to September / 2020)
}

\author{
Reem Al-Saadi Noora Aljaffali Anwar Qudaisat Jadulluh Al-ghazo* \\ Department of Dietetics - Hazem Mebaireek General Hospital (HMGH), Hamad Medical Corporation, Qatar
}

\begin{abstract}
There is limited information available describing the clinical and epidemiological features of COVID-19 patients at $(\mathrm{HMGH})$ in Qatar, also there is lack of reporting about temporal association of Malnutrition and severity of COVID-19. This study was aimed to describe the relationship between serum albumin levels and certain nutritional parameter with mortality rate for ICU-COVID-19 patients. The study was conducted retrospectively on a group of adult patients, who were admitted to ICU at HMGH, from 01/04/ 2020 to 30/09/ 2020, who's discharged from the hospital, whether recovered or expired, if they met the following inclusion criteria: ICU patients, $\geq 18$ years of age, laboratory confirmed positive of COVID-19. Data of ICU-COVID-19 patients at HMGH will be extracted from electronic medical record (EMR). Total of 1059 patients, among them $78(7.4 \%)$ patients were females and $981(92.6 \%)$ of them were males. Comparing with nationality $93(8.78 \%)$ patients were Qatari and 966(91.21\%) were non-Qatari, $951(89.8 \%)$ was survived \& $108(10.2 \%)$ were non survived, furthermore $12(11.1 \%)$ patients from the non-survived patients were Qatari \& $96(88.9 \%)$ were non-Qatari. In the results it found that $136(12.8 \%)$ of patients had Hyperlipidemia, 75 (7.1\%) had obesity, $577(54.5 \%)$ with diabetes, $513(48.41 \%)$ with hypertension, \& $129(12.2 \%)$ had chronic lung disease. 581 patients had albumin value below $35 \mathrm{gm} / \mathrm{L}$ on admission and 768 had below $35 \mathrm{gm} / \mathrm{L}$ before discharge. 478 patients had albumin level above $35 \mathrm{gm} / \mathrm{L}$ on admission and 291 patients had above $35 \mathrm{gm} / \mathrm{L}$ albumin level before discharge. Among nonsurvived patients who $108(100 \%)$ of them had low serum albumin levels before death and $80(74.1 \%)$ of them had below $35 \mathrm{gm} / \mathrm{L}$ albumin and 28 (25.9\%) patients had $35 \mathrm{gm} / \mathrm{L}$ and above albumin levels on admission. In Qatar there were adequate implementation of interventions, protections \& management to face COVID-19 prevention. Our study concluded that male patients \& hypoalbuminemia were significantly risk of mortality associated with COVID-19.
\end{abstract}

Keywords: hypoalbuminemia, nutritional parameter, mortality rate.

DOI: $10.7176 / \mathrm{FSQM} / 111-01$

Publication date:October $31^{\text {st }} 2021$

\section{Introduction}

COVID-19 consider as a new strain of Coronavirus, which start in Wuhan China in December 2019, and now become pandemic across the globe. The mortality rate of this virus over the world 3.4-4\%, which make more severe compared with flu, The elderly who have co-morbidity are succumbing more to the COVID-19, People with mild symptoms usually recover within 3 to 7 days, while those with previously have sever diseases take weeks to recover, the percentage rate of recovery for COVID-19 is expected to hit 90\% (WHO, 2020) $(1,2)$.

The COVID-19 disease in Qatar is part of the worldwide pandemic of corona virus disease 2019, according to the official reporting of ministry of public health (MOPH) the first confirmed case in Qatar was on 27 February 2020, relative to its population, Qatar ranks the highest number of confirmed cases compared to other countries of the world, and comes the second country after Saudi Arabia in the number of confirmed cases in compared to other middle east countries, The number of deaths in Qatar has been low, related to the reported of confirmed cases (3).

The mortality rate in Qatar based on governmental report and those from Johns Hopkins University is $0.3 \%$, which may be to the country's adequate healthcare system and the fact of most of residents are young and healthy, The State of Qatar early has taken all precautionary measures to prevent the spread of COVID-19, These precautionary measures can be changed based on the latest guidance of MOPH and Word health organization (WHO) regarding to the spread of COVID-19 (4).

COVID-19 has heterogeneous symptoms, many patients can show a wide array of symptoms while others remain carrier without symptomatic, most symptomatic patients vary from mild flu-like manifestations such as dry cough, myalgia, phlegm or diarrhea, to severe pneumonia or acute respiratory distress syndrome (ARDS), The exact pathobiology for severe and critically ill patients is still not clear (5).

Generally, the most of positive patients with COVID-19 have symptoms of respiratory infection, with mortality rate $1.4-4 \%$. The death in some cases that developed to critical illness may be due to massive alveolar damage and progressive respiratory failure, little is known regarding to the clinical markers for the ICU patients 
with covid-19 (6).

According to many studies, hypoalbuminemia may play a central role in the progression of symptoms from mild to severe COVID-19 especially those have malnutrition, and role in mortality rate across numerous clinical cases (7). Although the human serum albumin (HSA) has an antioxidant property, however its behavior and its relationship to the mortality rate in COVID-19 patients have not yet been studied (8).

The pathophysiology for hypoalbuminemia in certain co-morbidities (such as pancreatitis, burn, infection, trauma, and organ dysfunction) is secondary result, to increased capillary permeability, decreased protein synthesis, decreased half-life of serum albumin, increased volume of distribution, decreased serum albumin total mass, and increase expression of vascular endothelial growth factor, it has been supposed that, the pathophysiology for hypoalbuminemia is assassinated with hallmark of severe COVID-19 (9).

Human serum albumin level (HSAL) widely used in clinical practice, it reflects nutritional status and inflammatory response, which are related to outcomes of cancer treatment, and inflammatory diseases. Previous studies also found that HSAL was significantly lower in patients with severe COVID-19 (10).

In this study, we will hypothesize that, serum albumin levels, BMI with certain nutritional parameters (Vit D, Vit B12, HbA1c and Cholesterol) is significant at admission, which might reflect the severity of systemic inflammation, and this can serve as a predictive factor for COVID-19 outcomes. Our study will lead to the hypothesis that, lower HSAL may be used to identify patients at high risk of death in diagnosed patients.

According to the study conducted by Jiaofeng Huang, May 2020, the result showed that hypoalbuminemia is associated with the outcome of COVID - 19. Also, there is an inverse relationship between the level of albumin and increase the risk of death in COVID - 19 patients (9).

For Roberto de la Rica study conducted in Spain, it founds that Albumin might be of importance because of its association with disease severity in patients infected with

COVID-19 (5).

The objectives of the current study are:

1.To determine the relationship between the serum albumin level and nutritional parameters, with mortality rate of ICU-COVID-19 patients through admission period in ICU.

2. To compare the outcome of patients who have nutritional deficiencies with those patients without nutritional deficiencies through the admission period in ICU.

\section{Study Methodology}

The methodology adopted by the investigator is "quantitative Retrospective Data cohort study". The data was extracted from EMR after getting approval from the medical record department, all COVID-19 adult patients who were admitted to ICU at HMGH from 01/04/2020 to 30/09/2020, who's discharge from the hospital whether recovered or expired the researcher is assuming that the estimated sample size is 1000 .

\section{Study Population.}

The study, will be conducted retrospectively on a group of adult patients, with COVID-19 positive who were admitted to ICU at HMGH State of Qatar at (01/04-30/09/2020), whose discharged from the hospital whether recovered or expired, if they met the following inclusion criteria: ICU patients, $\geq 18$ years of age, laboratoryconfirmed diagnosis of COVID-19, through the admission period, \& they will not be followed after discharged from ICU. Also, the patients who were admitted to ICU with COVID-19 positive \& not discharged through the study period will be excluded.

\section{Data Collection \& Confidentiality}

Data was extracted from the Electronic medical record, data collection by reviewing the patient file electronically and confidentiality will be maintained. In our data collection sheet, we removed hospital number, names, and any other identifiers to protect patient confidentiality. We replaced the Health Card number with Serial number. Only the investigators have the access to study related files and the data will be documented in Excel file. The principal investigator and 3 co-investigators collected the data from the Cerner with the help of medical record. any link between the code and identifier was deleted after data collection and verification.

\section{Statistical Analysis.}

Descriptive statistics will be used to summarize and determine the sample characteristics and distribution of patients presented and diagnosed with COVID-19 infection. The normally distributed data and results will be reported with mean and standard deviation (SD) with corresponding 95\% confidence interval (CI); the remaining results will be reported with median and inter-quartile range (IQR). Categorical data will be summarized using frequencies and proportions. Associations between two or more qualitative will be assessed using Chi-square $(\chi 2)$ test or Fisher Exact test as appropriate. Quantitative data between the two independent groups will be analyzed using unpaired $t$ test. Data with a non-normal distribution will be compared using the Wilcoxon rank-sum test 
and presented as the median and interquartile range.

Baseline data including demographic, history of illness, clinical and laboratory variables will be included in the logistic regression analyses to assess their associations with COVID-19 severity and mortality. Univariate and multivariate logistic regression analyses will be carried out to determine the risk factors (using main exposure variables serum albumin level and nutritional parameters stated above) for severe COVID-19 mortality and results will be presented and reported in odds ratio (OR) and associated 95\% CI.. The optimal cutoff of the albumin level and nutritional parameters for predicting COVID-19 severity and mortality will be determined by analyses of the receiver operating characteristic (ROC) curve. Relationship between two quantitative variables will be examined using Pearson's or Spearman's correlation coefficients. All P values presented will be twotailed, and $\mathrm{P}$ values $<0.05$ will be considered as statistically significant. All Statistical analyses will be done using statistical packages SPSS 27.0 (SPSS Inc. Chicago, IL) software. The Statistical Package for the Social Sciences (SPSS 27.0 software) was used to analyses the data. Descriptive statistics was obtained using means (SD) and percentages. Several indicators were examined to determine the frequency and percentage of patients who have serum albumin level and nutritional parameters deficiencies related to mortality rate, for icu-covid-19 patients at Hazem Mebaireek general hospital (HMGH), state of Qatar (MARCH TO SEPTEMBER / 2020). P values less than 0.05 was considered as significant with confidence level $95 \%$.

\section{Results:}

The collected data from medical record were reviewed from 1st of April to end of September 2020 of patients who were diagnosed as COVID-19 positive \& admitted in ICU-HMGH, which included all patients in this period. Total of 1059, among them $78(7.4 \%)$ patients were females and $981(92.6 \%)$ of them were males. Comparing with nationality 93(8.78\%) patients were Qatari and 966(91.21\%) were non-Qatari, 951 (89.8\%) was survived \& $108(10.2 \%)$ were non survived, furthermore $12(11.1 \%)$ patient from the non-survived patients were Qatari \& 96 (88.9\%) were non-Qatari.

In the results it found that $136(12.8 \%)$ of patients had Hyperlipidemia, 75 (7.1\%) had obesity, $577(54.5 \%)$ with diabetes, 513 (48.41\%) with hypertension, \& 129 (12.2\%) had chronic lung disease, table (1).

Table (1): Results of Co-Morbidity with COVID-19 positive patients who were admitted to ICU

\begin{tabular}{|l|l|l|l|l|}
\hline Co-Morbidity & Yes & No & \\
\hline Hyperlipidemia & 136 & $12.80 \%$ & 923 & $87.20 \%$ \\
\hline Obesity & 75 & $7.10 \%$ & 984 & $92.90 \%$ \\
\hline Diabetes & 577 & $54.50 \%$ & 482 & 45.55 \\
\hline Hypertension & 513 & $48.40 \%$ & 549 & $51.60 \%$ \\
\hline Chronic lung disease & 129 & $12.20 \%$ & 930 & $87.80 \%$ \\
\hline
\end{tabular}

Results of nutritional parameters with COVID-19 positive patients who were admitted to ICU.

Table (2): Vitamin D value on admission and before discharge for non-survived patients.

\begin{tabular}{|l|l|l|}
\hline Reference value & On admission & before Discharge \\
\hline Below $30 \mathrm{ng} / \mathrm{mL}$ & 5 & 5 \\
\hline Above $30 \mathrm{ng} / \mathrm{mL}$ & 20 & 16 \\
\hline No result & 83 & 87 \\
\hline
\end{tabular}

Table (2) shows that on admission 5 patients had vitamin D value below $30 \mathrm{ng} / \mathrm{mL}, 20$ had above $30 \mathrm{ng} / \mathrm{mL}$ and 83 patients had no results. before discharge 5 patients had vitamin D value below $30 \mathrm{ng} / \mathrm{mL}, 16$ had above 30 $\mathrm{ng} / \mathrm{mL}$ and 87 patients had no results.

Table (3): Vitamin B12 value on admission and before discharge for non-survived patients.

\begin{tabular}{|l|l|l|}
\hline Reference value & On admission & before Discharge \\
\hline Below $133 \mathrm{p} \mathrm{mol} / \mathrm{L}$ & 0 & 0 \\
\hline Above $133 \mathrm{p} \mathrm{mol} / \mathrm{L}$ & 12 & 12 \\
\hline No result & 96 & 96 \\
\hline
\end{tabular}

Table (3 )shows the Vitamin B12 value among the patients during admission and before discharge, no patient reported below $133 \mathrm{ng} / \mathrm{mL}, 12$ patients had above $133 \mathrm{ng} / \mathrm{mL}$ of Vitamin B12 value on admission and before discharge, 96 patients had no result of Vitamin B12 value on admission and before discharge. 
Table (4): Cholesterol value on admission and before discharge for non-survived patients.

\begin{tabular}{|l|l|l|}
\hline Reference value & On Admission & Before Discharge \\
\hline Normal $<5.2 \mathrm{mmol} / \mathrm{L}$ & 30 & 31 \\
\hline Borderline high $5.2-6.2 \mathrm{mmol} / \mathrm{L}$ & 2 & 2 \\
\hline High value $>6.2 \mathrm{mmol} / \mathrm{L}$ & 3 & 2 \\
\hline No result & 73 & 73 \\
\hline
\end{tabular}

Table (4) cholesterol value on admission and before discharge, 30 patients had below $5.2 \mathrm{mmol} / \mathrm{L}$ on admission and 31 patients before discharge. 2 patients reported borderline high of cholesterol level $5.2-6.2 \mathrm{mmol} / \mathrm{L} \mathrm{on}$ admission and before discharge. 3 patients had high cholesterol level $>6.2 \mathrm{mmol} / \mathrm{L}$ on admission and 2 patients had high level before discharge. 73 patients had no results.

Table (5): $\mathrm{HbA} 1 \mathrm{c} \%$ value on admission and before discharge for non-survived patients.

\begin{tabular}{|l|l|l|}
\hline Reference Value & On Admission & Before Discharge \\
\hline Normal low $<4.8 \%$ & 0 & 0 \\
\hline Normal high $(4.8-6) \%$ & 11 & 11 \\
\hline High value $>6 \%$ & 45 & 45 \\
\hline No result & 52 & 52 \\
\hline
\end{tabular}

Table (5) shows that 0 patient reported the HbA1c value below 4.8\%, 11 patients had normal high level of HbA1c (4.8-6) \% on admission and before discharge, 45 patients had high level on admission and before discharge, 52 patients had no results.

Table (6): BMI value on admission and before discharge for non-survived patients.

\begin{tabular}{|l|l|l|}
\hline Reference value & Admission & Discharge \\
\hline Underweight value $<18.5 \mathrm{~kg} / \mathrm{m} 2$ & 2 & 2 \\
\hline Normal weight $18.5-25 \mathrm{~kg} / \mathrm{m} 2$ & 41 & 41 \\
\hline Overweight $25-30 \mathrm{~kg} / \mathrm{m} 2$ & 33 & 34 \\
\hline Obese $>30 \mathrm{~kg} / \mathrm{m} 2$ & 32 & 31 \\
\hline
\end{tabular}

Regarding BMI value the above table shows that 2 patients are underweight $<18.5 \mathrm{~kg} / \mathrm{m} 2$ on admission and before discharge, 41 patients had normal BMI on admission \& discharge, 33 patients are overweight on admission and 34 patient are overweight before discharge, and 32 patient are Obese $>30 \mathrm{~kg} / \mathrm{m} 2$ on admission \& 31 patient are Obese $>30 \mathrm{~kg} / \mathrm{m} 2$ before discharge.

Table (7): Albumin value on admission and before discharge for all ICU-COVID +ve patients:

\begin{tabular}{|l|l|l|}
\hline Reference value & On admission & Before discharge \\
\hline Below 35 gm/L & 581 & 768 \\
\hline Above 35 gm/L & 478 & 291 \\
\hline
\end{tabular}

Table (7) shows that 581 patients had albumin value below $35 \mathrm{gm} / \mathrm{L}$ on admission and 768 had below $35 \mathrm{gm} / \mathrm{L}$ before discharge. 478 patients had albumin level above $35 \mathrm{gm} / \mathrm{L}$ on admission and 291 patients had above 35 $\mathrm{gm} / \mathrm{L}$ albumin level before discharge.

Table 8: Albumin value on admission and before discharge for non-survived patients.

\begin{tabular}{|l|l|l|}
\hline Reference value & On admission & Before discharge \\
\hline Below 35 gm/L & $80(74.1 \%)$ & $108(100 \%)$ \\
\hline $35 \mathrm{gm} / \mathrm{L}$ and Above & $28(25.9 \%)$ & 0 \\
\hline
\end{tabular}

Table (8) shows that among non-survived patients who $108(100 \%)$ of them had low serum albumin levels before death and $80(74.1 \%)$ of them had below $35 \mathrm{gm} / \mathrm{L}$ albumin and $28(25.9 \%)$ patients had $35 \mathrm{gm} / \mathrm{L}$ and above albumin levels on admission.

\section{Discussion.}

On 30 January 2020, World Health Organization (WHO) announced that the COVID-19 outbreak was a Public Health Emergency of International Concern. As of 11 March, the WHO announced that COVID-19 is now declared as a pandemic. As of 31 March 2020, a total of 750,890 confirmed cases of COVID-19 have been reported in $>200$ countries. To date, most cases were reported in China, with some cases developing in other countries. The number of cases in China declined, during February 2020, while the number of cases reported outside China increased and the number of countries reporting cases increased. Several countries have demonstrated that COVID-19 transmission from one person to another can be slowed or stopped. Protection actions which followed in Qatar have saved lives and have provided the rest of the world with more time to prepare for the arrival of COVID- $19^{18}$. 
In 2019, the Ministry of Public Health worked with responsible persons to produced "Qatar National Preparedness and Response Plan for Communicable Diseases" to face the pandemic. the plan was comprehensive that embodies capacity building and readiness to manage potential outbreaks. The purpose of developing the response plan was to manage the threats of communicable diseases in terms of preventing their occurrence, mitigating the risks, controlling, and containing the disease that occurred. It also covers the management of different outbreak and pandemic scenarios. The plan was developed adapting the WHO's recommended Pandemic Influenza Risk Management Guide 2013 to support the harmonization of national and international pandemic preparedness and response ${ }^{19}$.

Despite these initial difficulties, death rates are low. The known mortality rate in Qatar based on government figures and those from Johns Hopkins University is $0.3 \%$, and mirrors those of smaller neighboring Gulf countries, which range from $0.1 \%$ (Kuwait) to Bahrain (0.6\%). Saudi Arabia's death rate, by contrast, is around 1.4\%. This places Qatar well below the World Health Organization's $3^{\text {rd }}$ March estimate of a 3.4\% global death rate. Although such figures are highly formative, most Gulf countries have demographic characteristics with a relatively small elderly population ${ }^{19}$.

The relationship between hypoalbuminemia with reduced survival may have several explanations, first acting as an anti-inflammatory and antioxidant protein, albumin may protect against the cytokine storm and the ensuing organ failure. It is of interest the inverse relationship between serum albumin and troponin levels, suggesting that albumin might exert some protection against myocardial injury. Second, albumin encompasses anticoagulant properties and inhibits oxidative stress- related clotting and platelet activation. Therefore the negative impact of hypoalbuminemia on clotting activation may be another factor accounting for poor survival this is suggested at least, in part by our findings of an inverse association between albumin and D-dimer levels, it is the marker of thrombotic risk and increased mortality in COVID-19. The study observation leads to the hypothesis that serum albumin analysis may be used to identify patients at higher risk of death in COVID-19 patients ${ }^{17}$.

Our study results shows that total of 1059 COV-D-19 positive patient, among them 78 (7.4\%) patients were females and 981(92.6\%) of them were males comparing to the study conducted by Mohitosh Biswas, COVID-19 male patients were associated with significantly increased risk of mortality compared to females ${ }^{13}$.

Present study also shows that among 1059 patients admitted in ICU in HMGH, 951(89.8\%) patients survived and $108(10.2 \%)$ patients died. Among 108 patients died, $96(88.9 \%)$ were males and $12(11.1 \%)$ were females, which is corelated to the study conducted by Mohitosh Biswas ${ }^{13}$, with result that COVID-19 male patients were associated with significantly increased risk of mortality compared to females.

In our study among 1059 patients, 581 (54.9\%) patients had albumin level below $35 \mathrm{gm} / \mathrm{L}$ on admission and 768 (72.5\%) had below $35 \mathrm{gm} / \mathrm{L}$ before discharge. 478 (45.1\%) patients had albumin level above $35 \mathrm{gm} / \mathrm{L}$ on admission and 291 (27.5\%) patients had above $35 \mathrm{gm} / \mathrm{L}$ albumin level before discharge. Among the 108 patients died, $28(25.9 \%)$ patients had serum albumin value above $35 \mathrm{gm} / \mathrm{L}$ on admission and $80(74.1 \%)$ patients had below $35 \mathrm{gm} / \mathrm{L}$ of serum albumin level on admission.

All the patients $108(100 \%)$ had low serum albumin level of less than $35 \mathrm{gm} / \mathrm{L}$ before death which is corelated to the study conducted by Dr. Ananya Mandal regarding the low albumin levels could predict poor outcome in COVId-19 patients. In this study of 48 patients, 21 were admitted into intensive care (ICU), and 27 were not. The symptoms prior to hospitalization included fever (in all patients), cough (in 85 percent patients), difficulty in breathing (in 76 percent patients), diarrhea (42 percent patients, and weakness (21 percent). The main reason for hospitalization among the patients was low blood oxygen (low SpO2 below 90 percent) and the presence of pneumonia in both lungs. Pneumonia in both lungs was seen in 94 percent of cases. Lower levels of blood albumin were significantly associated with poorer outcome and a longer stay in the hospital. The risk of death was also higher among those with lower levels of albumin in their blood ${ }^{15}$.

With this study the same study conducted by Muhammad Aziz, Rawish Fatima about the association of low serum albumin level with severe COVID-19 patients, which demonstrate the association of hypoalbuminemia and severe COVID-19. A low albumin level can potentially lead to early recognition of severe disease and assist clinicians in making informed decision for their patients ${ }^{16}$

A retrospective cohort study conducted by Roberto de la Rica, Marcio Borges, María Aranda, July 15, 2020 Low albumin levels are associated with poorer outcomes in a case series of COVID-19 patients in Spain, the objective of the study is to describe the clinical characteristics and epidemiological features of severe (non-ICU) and critically patients (ICU) with COVID-19 at triage, prior hospitalization. 48 patients (27 non-ICU and 21 ICU) resident in Mallorca, Spain (mean age, 66 years, (range, 33-88 years); 67\% males) with positive SARS-CoV-2 infection were analyzed. Lower albumin levels were associated with poor prognosis measured as longer hospital length and mortality. The study concluded that critically ill patients with COVID-19 present lymphopenia, hypoalbuminemia as well high levels of inflammation. Lower levels of albumin were associated with poorer outcomes in COVID-19 patients. Albumin might be of importance because of its association with disease severity in patients infected with SARS-CoV-2 ${ }^{17}$. 
Another study shows that Hypoalbuminemia predicts the outcome of COVID-19 independent of age and co-morbidity, conducted by Jiaofeng Huang 1, Aiguo Cheng reveals that Lower albumin level is seen in severe COVID-19 and is not parallel to the changes in alanine aminotransferase and aspartate aminotransferase levels. The study aimed to explore the impact of hypoalbuminemia in COVID-19. This retrospective cohort study included adult patients with confirmed COVID-19. The relationship between hypoalbuminemia and death was studied using binary logistic analysis. A total of 299 adult patients were included, $160(53.5 \%)$ were males and the average age was $53.4 \pm 16.7$ years. Approximately one-third of the patients had comorbidities ${ }^{20}$.

Xiaoyue Wang, Yan Xu study shows that the patients with mild and severe COVID-19 showed significant differences in terms of, pretreatment $C$-reactive protein-to-albumin ratio $(C A R)(P<0.05)$. The severity of COVID-19 was correlated positively with the comorbidity of CAR $(\mathrm{P}<0.05)^{21}$

From our study reports, in overall cases $577(54.50 \%$ ) patients had diabetes, and of the 108 expired patients 69 (63.9)\% had diabetes, which is similar to the study conducted by Aidin Rawshani shows that among persons with diabetes of either type COVID-19, related mortality is associated with older age, male sex, socioeconomic deprivation, non-white ethnicity, established cardiovascular disease, and impaired renal function, glycemic control and with both obesity and underweight.$^{14}$

Comorbidity may also relate to reduced immune function. For example, in diabetic patients, natural immune function reduced substantially which may restrict the body to produce respective antibody against any infection, Effects of various comorbidities such as hypertension, diabetes mellitus, respiratory disease, cardiovascular disease, cerebrovascular disease, kidney disease, liver disease, and cancer were compared between survivors and non-survivors with these comorbidities. All these comorbid conditions except liver disease were significantly higher in non-survivors compared to survivors ${ }^{13}$.

Our study shows that on admission 5 patients had vitamin D below $30 \mathrm{ng} / \mathrm{ml}, 20$ patients had above 30 $\mathrm{ng} / \mathrm{ml}$ and 83 patients had no results. Before death 5 patients had vitamin D below $30 \mathrm{ng} / \mathrm{ml}, 16$ patients had above $30 \mathrm{ng} / \mathrm{ml}$ and 87 patients had no results. Vitamin B12 among the patients during admission and discharge, no patient reported below $133 \mathrm{p} \mathrm{mol} / \mathrm{L}, 12$ patients had above $133 \mathrm{p} \mathrm{mol} / \mathrm{L}$ of Vitamin B12 on admission and discharge, 96 patients had no result of Vitamin B12 on admission and discharge. Nutrients play a vital role in the defense against infectious diseases and the regulation of inflammation; however, little is known with regards to COVID-19.

Obesity is a common chronic disease which affect other chronic diseases, and increases the risk of severe illness of COVID-19 ${ }^{23}$, according to CDC the obesity of adult persons with excess weight are at even greater risk during the COVID-19 pandemic; our study shows that from the 1059 cases $75(7.1 \%)$ were obese, of the 108 expired patients $31(28.7 \%)$ were obese.

Jae HyoungIm's study measured concentrations of vitamins B1, B6, B12, folate, vitamin D (25hydroxyvitamin D), selenium, and zinc in 50 patients with COVID-19. Vitamin D deficiency was shown in $76 \%$ of patients and selenium deficiency in $42 \%$. There was a significant difference compared to a control group of 150 people (vitamin D deficiency 43.3\%). Among 12 patients with respiratory distress, $11(91.7 \%)$ had one or more nutrient deficiency ${ }^{20}$.

Conclusion

In Qatar there were adequate Implementation of interventions, protections \& management to face COVID19 prevention. in our study, male patients \& hypoalbuminemia were significantly risk of mortality associated with COVID-19. Also, this study makes inquiries about the nutritional parameters' status in patients with COVID-19 and with the disease severity.

\section{Recommendation}

- Given Therapeutic management of albumin infusion for COVID-19 patients can decrease the mortality rate.

- To make blood investigation for the nutritional parameters for all patient with COVID -19, \& to follow the laboratory blood result.

- $\quad$ Further research is needed to evaluate the impact of nutritional deficiencies in patient with COVID-19.

\section{References}

1. Ryad Ghanam, aEdward L. Boone, b Abdel-Salam G. Abdel-Salamc, 2020, SEIRD MODEL FOR QATAR COVID-19 OUTBREAK: A CASE STUDY, Lett. Biomath., Vol. 0, Iss. 0 (0), pp. 1-10.

2. Xiaochen Li, Shuyun Xu, Muqing Yu, MD, 2020, Risk factors for severity and mortality in adult COVID-19 inpatients in Wuhan, Journal of Allergy and Clinical Immunology, Volume 146, Issue 1, July 2020, Pages $110-118$

3. Ministry of Public Health ,2020, Retrieved 26 "Coronavirus Disease 2019 - (COVID-9)", August 2020 , available fromhttps://www.moph.gov.qa/english/Pages/default.aspx .

4. Marc Owen Jones, April 2020, Covid-19 in Qatar: Well Versed in Crisis Management. Gulf Insights N 21 
5. Roberto de la Rica, Marcio Borges, Maria Aranda, et al, Published, 24 July 2020, Low Albumin Levels Are Associated with Poorer Outcomes in a Case Series of COVID-19 Patients in Spain: A Retrospective Cohort Study, Microorganisms 2020, 8, 1106.

6. Juyi Li, Meng Li, Shasha Zheng, et al, published online, 3 June 2020, Plasma albumin levels predict risk for nonsurvivors in critically ill patients with COVID-19, Biomark. Med. (Epub ahead of print)- ISSN 17520363.

7. Muhammad Aziz, Rawish Fatima, Wade Lee-Smithland Ragheb Assaly,2020, The association of low serum albumin level with severe COVID-19: a systematic review and meta-analysis. Aziz et al. Critical Care (2020) $24: 255$.

8. Francesco Violi, Roberto Cangemi, Giulio Francesco Romiti, et al, 2020, Is Albumin Predictor of Mortality in COVID-19? ANTIOXIDANTS \& REDOX SIGNALING Volume 00, Number 00, $2020{ }^{\text {a }}$ Mary Ann Liebert, Inc. DOI: 10.1089/ars.2020.8142.

9. Jiaofeng Huang, Aiguo Cheng, Rahul Kumar, et al, Accepted, 11 May 2020, Hypoalbuminemia predicts the outcome of COVID - 19 independent of age and co - morbidity, Journal of medical virology wiley. DOI: 10.1002/jmv.26003.

10. Xiaoyue Wang, Yan Xu huaian, Huang, Desheng Jiang, et al, Posted 08 Aug 2020, Increased pretreatment C-reactive protein-to albumin ratio predicts severe coronavirus disease 2019.Military medical research. DOI: 10.21203/rs.3.rs-31723/v21.

11. Lucie Allard I, Elise Ouedraogo, Julie Molleville, et al, 2020, Malnutrition percentage and association with prognosis in patients hospitalized for coronavirus disease 2019, Nutrients 2020, 12, 3679; doi:10.3390/nu12123679.

12. Zhong-Hua Wang, Ying-Wen Lin, Xue-biao Wei, et al, 2021, Predictive Value of Prognostic Nutritional Index on COVID-19 Severity, Front. Nutr. Front. Nutr, https://doi.org/10.3389/fnut.2020.582736.

13. Biswas M. a, Rahman S.a, Intervirology 2021, Association of Sex, Age, and Comorbidities with Mortality in COVID- 19 Patients: A Systematic Review and Meta-Analysis, https://doi.org/10.1159/0005125922021, Vol.64, No. 1.

14. Aidin Rawshani, Elin Allanson Kjölhede, Published April 30, 2021, Severe COVID-19 in people with type 1 and type 2 diabetes in Sweden: A nationwide retrospective cohort study DOI: https://doi.org/10.1016/j.lanepe.2021.100105, VOLUME 4, 100105, MAY 01, 2021.

15. Ananya Mandal, MD, May 13-2020, Low albumin levels could predict poor outcome in COVID-19, 2020.05.07.20094987; doi: https://doi.org/10.1101/2020.05.07.20094987.

16. Muhammad Aziz, Rawish Fatima, published 26 May 2020 the association of low serum albumin level with severe COVID-19: a systematic review and meta-analysis, Article number: 255 (2020).

17. Francesco Violi 1, Roberto Cangemi,2020, Is Albumin Predictor of Mortality in COVID-19 2020 Jun 22. doi: 10.1089/ars.2020.8142

18. Meike Janssen, Betty P. I. Chang, Hristo Hristov, et al, 2021, Changes in Food Consumption During the COVID-19 Pandemic: Analysis of Consumer Survey Data from the First Lockdown Period in Denmark, Germany, and Slovenia, https://doi.org/10.3389/fnut.2021.635859.

19. Edwin van Teijlingen, Brijesh Sathian, Padam Simkhada, Published online 2021 Jan 4, COVID-19 in Qatar: Ways forward in public health and treatment, Qatar Med J. 2020; 2020(3): 38

20. Jiaofeng Huang 1, Aiguo Cheng doi,2020, Hypoalbuminemia predicts the outcome of COVID-19 independent of age and co-morbidity: 10.1002/jmv.26003. Epub 2020 May 25

21. , Xiaoyue Wang, Yan Xu, Huang Huang, et al,2020, an increased pretreatment C-reactive protein-toalbumin ratio predicts severe novel coronavirus-infected pneumonia, 10.21203/rs.3.rs-31723/v1

22. , panel Jae HyoungIma, Young SooJe, November 2020, Nutritional status of patients with COVID-19, International Journal of Infectious Diseases, Volume 100, Pages 390-393

23. Obesity, Race/Ethnicity, and COVID-19, https://www.cdc.gov/obesity/data/obesity-and-covid-19.html 